\title{
MMW-HOTLINE
}

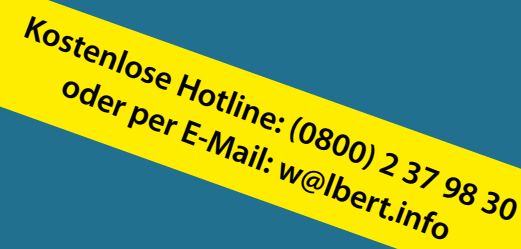

Leser der MMW können sich mit allen Fragen zur Abrechnung und Praxisführung an Helmut Walbert, Facharzt für Allgemeinmedizin, Würzburg, wenden. Sie erreichen ihn jeden Donnerstag von 13 bis $15 \mathrm{Uhr}$ unter der kostenlosen Rufnummer (0800) 2379830 oder per E-Mail: w@lbert.info.

\section{Wird der erhöhte Aufwand besonders vergütet?}

\author{
Frau H., MFA in Allgemeinarztpraxis \\ Dr. med. P. S., Hamburg:
}

Wir haben immer wieder Palliativpatienten zu versorgen. Kann der erhöhte Aufwand irgendwie gesondert verrechnet werden? Weiterhin hat sich einer unserer Patienten mit HIV infiziert und wird von uns betreut. Kann über die GOP 03212 hinaus hier der spezielle Aufwand irgendwie verrechnet werden?

Antwort: Ja! Seit dem 1.7.2009 gibt es die Möglichkeit, für die Behandlung/Betreuung von Palliativpatienten gegenüber den Krankenkassen Kostenpauschalen abzurechnen. Die Erbringung dieser Leistungen setzt- keine spezielle Weiter- oder Fortbildung voraus. Sie können in vorliegenden Fällen von jedem Hausarzt abgerechnet werden. Die Leistungen finden sich im Kapitel 1.4 „Besuche, Visiten, Prüfung der häuslichen Krankenpflege, Verordnung besonderer Behandlungsmaßnahmen ..." Es sind dies die GOP 01 425, „Erstverordnung der spezialisierten ambulanten Palliativversorgung ..." 25,06 € und die GOP 01 426, „Folgeverordnung zur Fortführung der spezialisierten ambulanten Palliativversorgung ...., höchstens zweimal im Behandlungsfall, 15,07 €. Diese Leistungen werden außerhalb des Regelleistungsvolumens und außerhalb der morbiditätsorientierten Gesamtvergütung bezahlt.

Bei der Betreuung von HIV-Patienten bleibt es bei der GOP 03 212. Es gibt zwar Zusatzpauschalen für die Behandlung von HIV-Patienten im EBM im Kapitel 30.10 "Leistungen der spezialisierten Versorgung HIV-infizierter Patienten“. Diese sind aber an die Genehmigung der zuständigen Kassenärztlichen Vereinigung gemäß der Qualitätssicherungsvereinbarung gekoppelt und setzen die entsprechende Qualifikation und eine Mindestpatientenzahl voraus. Damit sind sie spezialisierten Fachkollegen vorbehalten.

\section{Die KV hat mir die GOP 01820 gestrichen}

\section{Was haben wir falsch gemacht?}

\section{Dr. med. S. W., KV Sachsen:}

Die KV hat mir im Rahmen der sachlichen und rechnerischen Richtigstellung die Abrechnung der GOP 01820 neben der Versichertenpauschale gestrichen. Die GOP 01820 ist in der Präambel des Hausarztkapitels aufgeführt. Was haben wir falsch gemacht?

Antwort: Die GOP 01820 ist wie die GOP 01420 eine Vergütungsposition für eine Verwaltungsleistung mit entsprechend niedriger Vergütung: $€ 1,05$. Bei beiden GOP steht eine Abrechnungsbeschränkung, die die Streichung durch die KV begründet:„Die Gebührenordnungsposition ist nicht neben anderen Gebührenordnungspositionen und nicht mehrfach an demselben Tag berechnungsfähig." Da offensichtlich ein persönlicher Arzt-Patienten-Kontakt (APK) stattgefunden hat - es wurde die Versichertenpauschale (VP) abgerechnet -, streicht die KV die geringerwertige Leistung und vergütet die Versichertenpauschale. Der Vorgang ist vollkommen korrekt.

Dennoch sollte das Praxisteam weiterhin differenziert die erbrachten Leistungen erfassen und abrechnen. Der Leistungsinhalt der GOP 01820 gehört zu den „Sonstigen Hilfen", wird also außerhalb des Regelleistungsvolumens zu festem Punktwert und extrabudgetär vergütet. Diese Tatsache der zusätzlichen Vergütung sollte im Auge behalten werden und gegebenenfalls die VP zu einem späteren Zeitpunkt abgerechnet werden, wenn sich ein weiterer APK ergibt. Die Ausschlussbestimmungen der GOP 01430 „Die GOP ist im Arztfall nicht neben anderen GOP und nicht mehrfach an demselben Tag berechnungsfähig" und "Kommt in demselben Arztfall eine Versicherten-, Grund- und/oder Konsiliarpauschale zur Abrechnung, ist die GOP nicht berechnungsfähig." gelten für die GOP 01820 nicht und sichern somit die Abrechnungs- und Vergütungsfähigkeit. 